\title{
Prolonged hospitalisation for immigrants and high risk patients with positive smear pulmonary tuberculosis
}

\author{
R. Luzzati1 ${ }^{1}$, M. Confalonieri2, A. Cazzadori3, P. Della Loggia33, R. Cifaldi², \\ C. Fabris4, M. Biolo2, M. Borelli5, C. Longo2, E. Concia ${ }^{3}$
}

ABSTRACT: Prolonged hospitalisation for immigrants and high risk patients with positive smear pulmonary tuberculosis. R. Luzzati, M. Confalonieri, A. Cazzadori, P. Della Loggia, R. Cifaldi, C. Fabris, M. Biolo, M. Borelli, C. Longo, E. Concia.

Background and objective. Tuberculosis (TB) occurring in immigrants and resistance to drugs are major problems for TB control in Western countries. Directly observed therapy (DOT) reduces disease transmission, but this approach may have poor results among illegal immigrants. Our aim was to evaluate a prolonged hospitalisation programme to improve early outcome of TB treatment in high risk patients.

Methods. All the consecutive adult patients with sputum smear-positive pulmonary TB admitted to 2 Italian referral TB Centres were evaluated. Hospital-based DOT was provided to high risk patients up-to smear conversion. Demographic, microbiological and clinical conditions, as potential factors associated with confirmed smear conver- sion at 60 and 90 days of anti-tuberculous therapy were evaluated.

Results. 122 patients were studied, $45.9 \%$ of them were immigrants (20\% illegal) from high-prevalence TB countries. HIV testing was negative in all cases. Twelve patients had M. tuberculosis resistant to $\geq 1$ first-line anti-tuberculous agents. The rate of defaulting from TB treatment was $7.3 \%$. Sputum smear became negative in $84.4 \%$ cases after 60 days and $93.3 \%$ cases after 90 days. At such time, smear conversion rates were similar among different high risk subgroups such as illegal immigrants $(\mathbf{9 5 . 9 \%})$, legal foreign-born $(\mathbf{9 2 . 5 \%})$ and Italian persons (94.8\%). Persistent sputum smear positivity was independently correlated with the extent of pulmonary lesions at $60(p<0.0001)$ and 90 days $(p=0.038)$ of hospital-based DOT.

Conclusions. These findings suggest that prolonged hospitalisation for illegal immigrants and high risk TB patients, may positively influence the early outcome of TB treatment despite of drug resistance and legal status.

Monaldi Arch Chest Dis 2011; 75: 2, 141-145.

Keywords: Pulmonary tuberculosis, Mycobacterium tuberculosis, Drug-resistant tuberculosis, Sputum smear conversion, hospitalization, Directly observed therapy, Illegal immigrants.

1 Infectious Diseases Unit, University Hospital, Trieste,

2 Department of Pulmonology, University Hospital, Trieste,

3 Infectious Diseases Unit, University Hospital, Verona,

4 Department of Microbiology, University Hospital, Trieste,

5 Department of Mathematics and Computer Science, University of Trieste, Italy.

Correspondence: Marco Confalonieri MD, Dept. Pneumology, University Hospital, Strada di Fiume 447, 34149 Trieste, Italy; e-mail: marco.confalonieri@aots.sanita.fvg.it

\section{Introduction}

Increased proportion of tuberculosis (TB) occurring among immigrants and resistance to drugs are major problems for $\mathrm{TB}$ control in Western countries including Europe and United States [1, 2]. A high rate of defaulting from treatment among high risk patients is a major task to optimise TB control in low incidence countries [3]. Early detection and treatment by directly observed therapy (DOT) reduces disease transmission [4], but active therapy obtained a low completion rate among illegal immigrants according to a previous study [5]. Sputum smear conversion at the second month of treatment is a key result to determine TB control [6], but this early outcome depends on a strict adherence to treatment. The need for efficient care delivery led us to establish the strategy of long hospital stay in order to manage the high risk of positive smear pulmonary TB patients because of their social status and/or drug resistance. The aim of this 4- year duration study was to evaluate the effectiveness of our hospital-based strategy to achieve sputum smear conversion after 60 and 90 days from the initiation of hospital-based DOT.

\section{Methods}

This study included all consecutive adult patients (aged $>18$ years) admitted to two referral TB Centres (University Hospital of Trieste and Verona, Italy) for high risk positive smear culture-confirmed pulmonary TB between January 2004 and December 2008. Both TB centres are located in 2 metropolitan areas in northern east Italy (Trieste and Verona), with a population of nearly 250,000 and 700,000 respectively. The decision to admit to hospital a sputum smear positive pulmonary TB patient for a hospital-based DOT treatment was taken according to at least one of the following criteria: a) resistance to one or more anti-TB drugs, b) foreignborn person, c) illegal immigrant, d) previous treat- 
ment, e) being an injecting drug user, f) HIV infection, g) social and/or familiar condition not assuring good adherence to treatment (living alone, residents of confined institutions, comprehension difficulties on modalities of treatment). Decision on patient hospital discharge was taken when the patient achieved into clinical improvement and confirmed sputum conversion following hospital-based DOT. The sputum was collected on a 15 -day period basis until the smear became negative for 3 consecutive samples (confirmed smear conversion), which were collected on different days. The laboratories performed culture and drug sensitivity test including second-line drugs, and were validated according to the World Health Organization External Quality Assessment WHO EQA) proficiency scheme, being part of the Multicenter Italian Study on Resistance to Antituberculosis Drugs (SMIRA) network [7]. Multidrug-resistant (MDR) TB is defined as a specific form of drug-resistant TB due to TB bacteria resistant to at least isoniazid and rifampicin. Cases with follow-up shorter than 60 days were excluded. Analyses focused on potential factors associated with confirmed smear conversion at 60 and 90 days of anti-tuberculous therapy. Candidate variables were: age, sex, country of origin (Italy or other countries), illegal immigrant condition, underlying diseases (chronic obstructive pulmonary disease, cancer, diabetes mellitus, alcohol abuse, and HIV infection), previous history of tuberculosis, semiquantitative evaluation of organisms on sputum smear ( $\leq$ or $>1 / 10$ fields) [8], resistance to any firstline antituberculous drug, presenting pulmonary $\mathrm{X}$ ray pattern (cavitary or not cavitary disease) and disease extent. Pulmonary disease extent on the initial chest radiography was based on the number of lung zones involved; each lung was considered to have 3 zones (upper, middle, and lower). Involvement of 1-2 zones was considered to be localized disease, 3-4 zones as moderate disease, and 5-6 zones as extensive disease [9]. A rough comparison of the results of this study with estimates of the costs of prolonged hospitalisation and drop-out rate of historically patients was finally made.

\section{Statistical analysis}

Normally distributed data was tested by Kolmogorov-Smirnov, and was summarised by mean (median) and standard deviation (quartiles). Statistical inference in the former case was approached by $F$ test and subsequently by Student-Welch $t$ test, whereas in the latter by Wilcoxon test. Survival analysis was applied to determine the difference in time to event data. Generalised linear models with binomial error distribution and logistical linking were used to assess covariate predictors involved in smear negativity. A logistic model was performed to check for independent risk factor(s). In all instances, alpha error $=5 \%$. To assess covariate associated to smear conversion, a multivariate additive generalized linear model with binomial (positive vs. negative) response was analysed. A significant minimal adequate models fitting the data were found by backward elimination process. Statistical analysis was performed by open source statistical package $\mathrm{R}$ version 2.6.2 ( $\mathrm{R}$ Development Core Team: R: A language and environment for statistical computing. R Foundation for Statistical Computing, Vienna, Austria. http://www.R-project.org).

\section{Results}

Among a total of 228 patients admitted to our hospitals for pulmonary TB during the study period, 122 patients, $76(62.3 \%)$ males and 46 females, had sputum smear-positive culture-confirmed TB filling one or more criteria for high risk smear positive TB. A total of 7,13, and 18 patients were discharged from hospital during the follow-up respectively within 15day, 60-day, and 90-day periods after the initiation of anti-tuberculous therapy. The reasons for discharge varied (family, job, personal decision, etc.), but only 15 patients were lost at follow-up. The rate of defaulting from TB treatment was $7.3 \%$ (9 patients), and 1 of them did not have confirmed smear conversion. Underlying diseases were present in $36 \%$ of cases, and included chronic obstructive pulmonary disease (16 cases), cancer (15 cases) and diabetes (13 cases). Twelve patients $(9.8 \%)$ had $M$. tuberculosis bacilli resistant to $\geq 1$ first-line anti-tuberculous agents. No second-line drug resistance was detected. Of the latter patients, one had a multidrug-resistant isolate (MDR). Fifty-six patients $(45.9 \%)$ were immigrants and refugees from high-prevalence countries. Twenty-four patients $(20 \%)$ were illegal immigrants. Twenty patients $(16.4 \%)$ had a medical history of alcohol abuse with wide range of liver and neurologic involvement. Human Immunodeficiency Virus (HIV) testing was negative in all cases, and 3 patients were injecting drug user. Adherence to treatment was $96 \%$ among patients who completed the 60 and 90 days hospital-based DOT period. The treatment completion rate was $93.4 \%$ including the patients lost at follow-up. Hospital discharge following confirmation of sputum smear conversion was obtained after a mean of $85 \pm 12$ days. At that time, all patients started to receive the current outpatient DOT programme. Sputum examination of the smear became negative in $25(21.8 \%)$ cases after 15 day of treatment, $60(53.1 \%)$ cases after 30 days, $92(84.4 \%)$ cases after 60 days and $97(93.3 \%)$ cases after 90 days. Culture of sputum for M. tuberculosis became negative in $9(7.8 \%), 32(27.8 \%), 59(54.1 \%), 73$ $(70.2 \%)$ cases respectively after $15,30,60,90$ days of treatment. Smear conversion rates after 90 days were similar among high risk subgroups: illegal immigrants (95.9\%), legal immigrants $(92.5 \%)$ and Italian persons $(94.8 \%)$, with no statistical difference Factors potentially associated with smear conversion at 60 and 90 days of treatment are detailed in table I. By univariate analysis, cavitary pulmonary disease, moderate or extensive X-ray pulmonary lesions, and resistance to any antituberculous drug resulted to be significantly associated with persistent smear positivity. To assess covariate associated to smear conversion, an additive generalised linear model with binomial (positive vs. negative) response was analysed. Two significant minimal adequate models fitting the data were found by backward elim- 
ination process, as follows: at 60 days, moderate or extensive pulmonary lesions $(p<0.0001)$; at 90 days, antibiotic resistance to any first-line anti-tuberculous drug ( $p=0.007)$ and moderate or extensive pulmonary lesions ( $p=0.038)$. Log-rank testing showed high significant difference $(p<0.001)$ between time to smear conversion only to curves belonging to patients with moderate or extensive pulmonary lesions on one hand, and localised lesions on the other (figure 1).

No association was found between sputum conversion at 60-90 days from hospital-based DOT initiation and the condition of illegal immigrant and/or legal immigrant, age, gender, presence of cavitary lesions, co-morbidities, sputum hypersecretion ( $>20 \mathrm{~mL} /$ day), drug-resistance (intercept $\mathrm{z}$ value $=-2.160)$.

Estimate of the costs of prolonged hospitalization and previous failure/drop-out rate among illegal immigrants

The average cost of hospitalisation at our Infectious Diseases Departments is presumed by our Administration to average $€ 440.00$ per day. The mean previous duration of hospital stay for active TB was $20 \pm 9$ days. The prolonged hospitalisation programme for high risk patients with sputum smear positive TB resulted in a duration of hospital stay longer than before $(98 \pm 18$ days $)$, so the extra hospital stay is $78 \pm 9$ days. The extra hospi- tal costs of such a prolonged hospitalisation may be roughly estimated in $€ 34320,00 \pm € 3960,00$. On the other hand, the revision of the historical data (2000-2003) from our registry showed that the rate of drop-out of TB treatment among Italian high risk TB patients was higher (20-25\%), and among illegal immigrants was even much higher (40-45\%) before the program of prolonged hospitalization than after to implement it.

\section{Discussion}

In our series, a prolonged hospitalisation programme allowed to achieve efficient care delivery in high risk smear positive pulmonary TB patients, including illegal immigrants. In fact, sputum smear conversion was very high (up to $94 \%$ ) after 90 days of DOT among these TB patients usually known to be at high risk for treatment failure (drug resistance, socially marginalised groups, illegal immigrants) $[10,11]$. This finding can be due to various factors including the absence of HIV-infected patients and the quite low rate of defaulting from treatment $(7.3 \%)$ in our patient population. The aim of tuberculosis treatment is to obtain a high cure rate and a low default rate. This is mainly a matter of management of the patients. It has been demonstrated long time ago that it is quite possible to reach this target on an ambulatory base, but this can be very difficult to obtain for illegal immigrants and other high risk

Table 1. - Potential factors associated with sputum smear conversion at 60 and 90 days of antiTB therapy

\begin{tabular}{|c|c|c|c|c|c|c|}
\hline \multirow[b]{2}{*}{ Variables } & \multicolumn{2}{|c|}{ After 60 days hospital-based DOT } & \multicolumn{4}{|c|}{ After 90 days hospital-based DOT } \\
\hline & Smear-negative cases & Smear-positive cases & $p$ & Smear-negative cases & Smear-positive cases & $p$ \\
\hline \multirow[t]{3}{*}{ Age: } & 27.50 & 34.25 & 0.21 & 29.00 & 34.50 & 0.49 \\
\hline & 40.00 & 47.50 & & 40.00 & 41.00 & \\
\hline & 68.50 & 72.00 & & 68.00 & 65.00 & \\
\hline \multirow{2}{*}{ Sex: } & 55 & 13 & 0.34 & 59 & 6 & 0.19 \\
\hline & 36 & 5 & & 38 & 1 & \\
\hline \multirow[t]{2}{*}{ Nationality: } & 45 & 11 & 0.38 & 46 & 4 & 0.62 \\
\hline & 47 & 7 & & 51 & 3 & \\
\hline \multirow[t]{2}{*}{ Underlying diseases: } & 38 & 4 & 0.11 & 39 & 1 & 0.17 \\
\hline & 53 & 14 & & 58 & 6 & \\
\hline New TB case & 74 & 14 & 0.71 & 80 & 6 & 0.83 \\
\hline Relapsing TB case & 17 & 4 & & 17 & 1 & \\
\hline \multicolumn{7}{|l|}{ Sputum quantification: } \\
\hline \multirow{2}{*}{$\begin{array}{l}<1 / 10 \text { fields } \\
\geq 1 / 10 \text { fields }\end{array}$} & 19 & 4 & 0.18 & 21 & 1 & 0.22 \\
\hline & 26 & 12 & & 28 & 5 & \\
\hline \multirow[t]{2}{*}{ Cavitary disease: } & 49 & 14 & 0.06 & 53 & 5 & 0.39 \\
\hline & 42 & 4 & & 44 & 2 & \\
\hline \multirow{2}{*}{$\begin{array}{l}\text { X-ray lesion extent: localised } \\
\text { moderate-extensive }\end{array}$} & 47 & 2 & 0.001 & 50 & 1 & 0.048 \\
\hline & 44 & 16 & & 45 & 6 & \\
\hline \multirow[t]{2}{*}{ Resistance to any drug: } & 9 & 3 & 0.17 & 9 & 3 & 0.007 \\
\hline & 82 & 15 & & 88 & 4 & \\
\hline
\end{tabular}




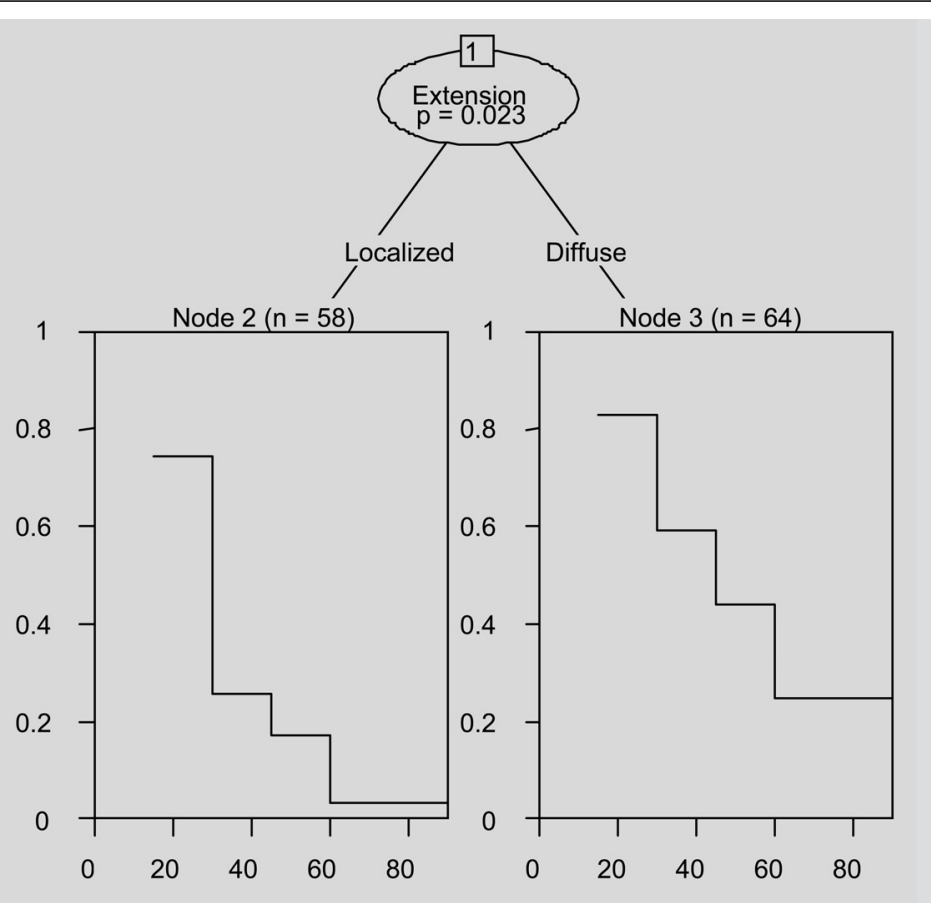

Fig. 1. The two edges of this recursive partitioning conditional non-parametric inference tree show that the localized vs. non-localized (moderate or wide amplitude) Rx extension lesions is a significant predictor $(\mathrm{p}=0.023)$ of the time to smear conversion. No other predictor of 90 days smear conversion was found.

TB patients. Sputum smear conversion at the second month of anti-tuberculous therapy has been considered a predictor of treatment success for pulmonary TB [6], and non-compliance with therapy is associated with poor treatment outcome, acquired drug resistance and increased risk of relapse [10, 11]. The European case-definition of TB is based on culture, and culture conversion is considered as informative as the sputum smear conversion [12]. The encouraging results of our prolonged hospital-based DOT approach were confirmed even in illegal immigrants, which represent a particularly difficult population to achieve compliance to treatment for any TB program in Italy $[5,13]$. Almost a half of our study population was foreign-born people, illegal immigrants being more than half of them. Adherence to treatment was high among hospitalised illegal immigrants with a quite high smear conversion rate after 90 days $(95 \%)$ considering their difficulty to comply with the treatment. Also the results of culture conversion were very similar to smear conversion, this is important because of the risks in the community of sputum conversion only [14]. Our findings might encourage to carry out novel health policies towards such difficult to care TB patients, whose burden on TB control is increasing in low prevalence Countries. The prolonged hospitalisation due to respiratory isolation of these patients may contribute to hasten the spreading of TB resistance and positive smear contacts among general population. Limited data is available to predict the length of time required for a patient to achieve sputum conversion after starting therapy for pulmonary tuberculosis. Although data suggests that once a patient receives effective treatment, infectiousness rapidly ceases [15], the Centers for Disease Control and Prevention (CDC) still recom- mend the suspension of respiratory isolation only when patients are receiving effective chemotherapy, their clinical conditions are improving, and when 3 consecutive sputum samples, collected on different days, are AFB-smear-negative [17]. Nevertheless, the closure of sanatorium hospitals in low prevalence Countries made hospitalization less frequent and hospital stay shorter than in the past. A major cause of the resurgence of TB in Western Countries is the increased proportion of immigrants from countries where TB is endemic [17]. Furthermore, the condition of illegal immigrant is associated with severe socioeconomic outcasting, uncertain working and living conditions, high mobility and, perhaps, the perception of TB therapy as much less essential than more immediate needs together with fear of deportation. DOT for illegal immigrants with TB requires more human and financial resources than for other patients. Previous Italian surveys already reported a significant correlation between compliance with treatment and legal citizenship status [18]. Even if DOT is expected to increase the rates of completion of treatment $[19,20]$, the pooled results of randomised controlled trials provide no assurance that current DOT has any quantitatively important effect on cure or treatment completion in people receiving treatment for $\mathrm{TB}$, a recent Cochrane review stated [21]. Providing an hospitalbased optimal adherence to treatment, we find that to be a foreign-born people and/or an illegal immigrant does not represent an independent risk factor for smear conversion failure. In our study population, the only variable independently associated with persistence of smear positivity during such period of treatment was the extent of pulmonary lesions. Several factors including numerous AFB on the initial smear and cavitary pulmonary disease 
were reported to be associated with sputum smear conversion at nearly 30-90 days of anti-tuberculous therapy [23-26]. We have found that cavitary disease was associated with persistently smear positive for AFB at 60 days of treatment in bivariate analysis. It became apparent on an additive statistical analysis, however, that cavitary disease was not and independent predictor in our series of high risk patients on prolonged hospital-based DOT. Although costs of prolonged hospitalisation are quite high, the overall costs of defaulting might be higher [22], but this issue was not an end-point of our study. We estimated an extra-cost of hospitalisation of more than $€ 30,000.00$ per patient. Nevertheless, the rate of drop-out and failure of TB treatment resulted much lower after the implementation of a prolonged hospitalisation programme than before. Finally, it must be clearly stated that the present study has several limitations: the study design is retrospective, a control group is lacking, a calculation of the costs of such a prolonged hospitalisation was not provided.

In conclusion, our findings suggest that a prolonged hospital-based DOT programme can obtain quite favourable early outcome in high risk patients including foreign-born and illegal immigrants. If this accounts for a better TB control with compatible cost in the general population of low incidence countries remain to be shown. Further prospective studies are needed to determine the cost-effectiveness of this therapeutic approach.

\section{References}

1. Rieder HL, Zellweger JP, Raviglione MC, Keizer ST, Migliori GB. Tuberculosis control and international migration in Europe. Eur Respir J 1994; 7: 1545-1553.

2. Centers for Disease Control and Prevention. Tuberculosis morbidity among US-born and foreign-born populations - United States, 2000. MMWR Morbid Mortal Wkly Rep 2002; 51: 101-4.

3. Sumartojo E. When tuberculosis treatment fails: a social and behavioral account of patient adherence. $\mathrm{Am}$ Rev Resp Dis. 1993; 147: 1311-20.

4. Weis SE, Slocum PC, Blais FX, et al. The effect of directly observed therapy on the rates of drug resistance and relapse in tuberculosis. N Engl J Med 1994; 330: 1179-1184.

5. Matteelli A, Casalini C, Raviglione MC, et al. Supervised preventive therapy for latent Tuberculosis infection in illegal immigrants in Italy. Am J Respir Crit Care Med 2000; 162: 1652-1655.

6. Feng-Zeng Z, Levy MH, Sumin W. Sputum microscopy results at two and three months predict outcome of tuberculosis treatment. Int J Tuberc Lung Dis 1997; 1: 570-572.

7. Migliori GB, Centis R, Fattorini L, Besozzi G, Saltini C, Orefici G, Piersimoni C, Gori A, Cassone A, Italian Multicentre Study on Resistance to Antituberculosis drugs (SMIRA) Study Group. Monitoring the quality of laboratories and the prevalence of resistance to antituberculosis drugs: Italy, 1998-2000. Eur Respir J 2003; 21: 129-34.

8. American Thoracic Society and the Centers for Disease Control and Prevention: Diagnostic Standards and Classification of Tuberculosis in Adults and Children. Am J Respir Crit Care Med 2000; 161: 1376-1395.

9. Al-Moamary MS, Black W, Bessuille E, Elwood RK, Vedal S. The significance of the persistent presence of acid-fast bacilli in sputum smears in pulmonary tuberculosis. Chest 1999; 116: 726-731.

10. Pablos-Mendez A, Knirsch CA, Barr RG, Lerner BH,
Frieden TR. Nonadherence in tuberculosis treatment: predictors and consequences in New York City. Am J Med 1997; 102: 164-170.

11. Caylà JA, Rodrigo T, Juan Ruiz-Manzano J, Caminero JA, Vidal R, José M García JM, Blanquer R, Casals M, and the Working Group on Completion of Tuberculosis Treatment in Spain (Study ECUTTE). Tuberculosis treatment adherence and fatality in Spain. Respiratory Research 2009; 10: 121.

12. Rieder HL, Watson JM, Raviglione MC, et al. Surveillance of tuberculosis in Europe. Working Group of the World Health Organization (WHO) and the European Region of the International Union Against Tuberculosis and Lung Disease (IUATLD) for uniform reporting on tuberculosis cases. Eur Respir J 1996; 9: 1097-104.

13. Centis R, Migliori GB, and Tuberculosis Study Group. National A.I.P.O. (Italian Association of Hospital Pneumologists); SMIRA Group (Multicentre Italian Study on Drug Resistance); National Tuberculosis Project, Istituto Superiore di Sanità. Evaluation of tuberculosis treatment results in Italy, report 1999. Monaldi Arch Chest Dis 2002; 57: 297-305.

14. Kruijshaar ME, Watson JM, Drobniewski F, et al. Increasing antituberculosis drug resistance in the United Kingdom: analysis of National Surveillance Data. BMJ 2008; 336: 1231-4.

15. Ritchie SR, Harrison AC, Vaughan RH, Calder L, Morris AJ. New recommendations for duration of respiratory isolation based on time to detect Mycobacterium tuberculosis in liquid culture. Eur Respir J 2007; 30: 501-07.

16. Centers for Disease Control and Prevention guidelines for preventing the transmission of Mycobacterium tuberculosis in health-care settings, 2005. MMWR 2005; 54: 1-141.

17. Scotto G, Fornabaio C, Prato R, Saracino A, Tartaglia A, Di Tullio R, Carbonara S, Angarano G, Italian Study Group for Infectious Diseases in Immigrants. Tuberculosis and immigrants: a SIMIT (Italian Society of Infectious Diseases) Clinical, Epidemiological Multicentric Research Investigation. New Microbiol 2009; 32: 39-47.

18. Carvalho AC, Migliori GB, Cirillo DM. Tuberculosis in Europe: a problem of drug resistance or much more? Expert Rev Respir Med 2010; 4: 189-200.

19. Global Tuberculosis Programme. Treatment of tuberculosis. Geneva, CH. 1997 WHO Report: WHO/TB/1997: 220.

20. Rieder HL. Sputum smear conversion during directly observed treatment for tuberculosis. Tubercle Lung Dis 1996; 77: 12429.

21. Volmink, Garner. Directly observed therapy for treating tuberculosis. Cochrane Database Syst Rev 2007; 17: CD003343.

22. Borgdorff MW, Veen J, Kalisvaart NA, J.F. Broekmans JF, Nagelkerke NJD. Defaulting from tuberculosis treatment in the Netherlands: rates, risk factors and trend in the period 1993-1997. Eur Respir J 2000; 16: 209-213.

23. Telzak EE, Fazal BA, Pollard CL, Turett GS, Justman JE, Blum S. Factors influencing time to sputum conversion among patients with smear-positive pulmonary tuberculosis. Clin Infect Dis 1997; 25: 666-670.

24. Singla R, Osman MM, Khan N, Al-Sharif N, Al-Sayegh MO, Shaikh MA. Factors predicting persistent sputum smear positivity among pulmonary tuberculosis patients 2 months after treatment. Int J Tuberc Lung Dis 2003; 7: 58-64.

25. Dominguez-Castellano A, Muniain MA, RodriguezBano J, et al. Factors associated with time to sputum smear conversion in active pulmonary tuberculosis. Int J Tuberc Lung Dis 2003; 7: 432-38.

26. Guler M, Unsal E, Dursun B, Aydln O, Capan N. Factors influencing sputum smear and culture conversion time among patients with new case pulmonary tuberculosis. Int J Clin Pract 2007; 61: 231-235. 